\title{
Morphology of semicrystalline diblock copolymer thin films upon directional solvent vapor flow
}

Ezzeldin Metwalli ${ }^{1}$, Jan Perlich ${ }^{1,2}$, Weinan Wang ${ }^{1}$, Alexander Diethert ${ }^{1}$, Stephan V. Roth ${ }^{2}$, Christine M. Papadakis ${ }^{1}$, and Peter Müller-Buschbaum ${ }^{1, *}$

${ }^{1}$ Physik-Department LS E13, TU München, James-Franck-Strasse 1, 85747 Garching (Germany)

${ }^{2}$ HASYLAB at DESY, Notkestr. 85, 22603 Hamburg (Germany)

[*] Tel: +49(0)8928912451, Fax: +49(0)8928912473, E-mail: muellerb@ph.tum.de

\begin{abstract}
The possibility of achieving templates of highly ordered nanostructures with crystalline nanoobjects embedded inside an amorphous matrix is addressed. Atomic force microscopy and grazing incidence small angle X-ray scattering (GISAXS) are used to probe the morphology at the surface and inside thin films of the diblock copolymer polystyrene-block-poly(ethylene oxide) $\mathrm{P}(\mathrm{S}-b$-EO). Directional benzene vapor flow introduces the orientation of PEO cylinders inside the PS matrix. Within a limited film thickness regime, the cylinders are oriented parallel to the sample surface in domains of several micrometers due to shearing effects caused by the flow of benzene vapor in parallel to the polymer surface. The crystallization of the PEO block by the benzene vapor treatment is also observed and crystals with sizes that approach that of the confined PEO domains are formed. Perpendicular orientation of the PEO crystalline stems with respect to the PEO cylindrical nano-domains is deduced with grazing incidence X-ray diffraction (GIXRD). X-ray reflectometry and scanning electron microscopy (SEM) complement the investigation.
\end{abstract}

\section{Keywords}

block copolymer, morphology alignment, self-assembly, grazing incidence small-angle X-ray scattering (GISAXS), semicrystalline polymers 


\section{Introduction}

In recent decades diblock copolymers (DBCs) have attracted immense attention for their role in nanotechnology applications. ${ }^{[1]}$ The ability of DBCs to create ordered nanostructures, as well as the ease with which they can be processed at low cost, open potential opportunities in surface patterning as a replacement for the time-consuming and costly conventional lithographic technique. ${ }^{[2,3]}$ Moreover, the soft nano-structured DBC film can also be used as a template for patterning of hard inorganic materials, such as metal nanoparticles. ${ }^{[4-11]}$ The chemically distinct and immiscible polymer blocks of the DBCs go through a self-assembly process during the microphase separation forming an ordered pattern on a scale of nanometers. An expedient tunability of the size, shape, and periodicity of the microphase-separated domains is achieved by manipulating the DBCs' molecular characteristics. A fine control over long-range ordering of the microdomain structures allows the fabrication of integrated microand nano-systems with a high degree of complexity and functionality. ${ }^{[12-14]}$ However, the selfassembly process generally produces structures that lack long-rang order with a population of uncontrolled defects. ${ }^{[15,16]}$ Thus currently, it is still a challenge to produce highly ordered DBC nano-domains over large areas with controlled and preferred orientation of the blocks. Therefore, directional self-assembly is a must for precise control over the alignment of the emerged morphologies. So far, several techniques have been successful in achieving an enhanced morphology alignment of DBCs by precisely localizing the nano-sized domains; these have included chemical, ${ }^{[17,18]}$ and topographical ${ }^{[15]}$ patterned surfaces as well as external fields, viz., surface, ${ }^{[19,20]}$ mechanical, ${ }^{[21]}$ electric, ${ }^{[22]}$ and solvent evaporation. ${ }^{[16,23-26]}$ However, mostly DBCs with two amorphous blocks were mainly focused on and less attention was paid to DBCs with one crystalline block. Possible crystallization of one block can inhibit the development of highly ordered micro-phase separation structures. Moreover, diblock copolymers with one crystalline block offer the possibility to achieve crystalline nano-objects in a matrix of amorphous polymer. Such types of templates are highly promising for applications in advanced electronics based on polymer templates.

Recently, Russell and coworkers ${ }^{[23]}$ have employed a unidirectional solvent evaporation method that helps the self-assembly process of the DBC to be directed normal to the film surface. During the slow evaporation stage, the initial order at the polymer surface gradually propagates from the polymer-vapor interface to the polymer-surface interface, thereby creating highly ordered and preferentially oriented cylinder morphology of the DBC. In the latter study, ${ }^{[23]}$ evaporation in the solvent annealing step was employed to be oriented vertically with respect to sample surface. 
In the present investigation, we focus on a DBC system with one crystalline and one amorphous block. We used polystyrene-block-poly(ethylene oxide) DBC films, denoted $P(S-$ $b-E O)$ that forms cylinder morphology with parallel orientation relative to the surface plane. Poly(ethylene oxide) based diblock copolymer is important material for applications related to solid-state batteries and fuel cells. The PEO blocks in DBCs is the polymer segment that can solvate and accommodate lithium ions for nano-structured polymer membrane with high ionic conductivity. ${ }^{[27]}$ Here, we perform a novel type of solvent vapor treatment of the spin-coated cylinder forming DBC film to achieve highly ordered nano-patterns. It is based on solvent vapor flowing parallel to the polymer film surface. An induced crystallization via vapor treatment of the polymer film is observed for all film thicknesses.

\section{Experimental}

Substrate cleaning and thin film preparation: Glass substrates $\left(2.5 \times 7.5 \mathrm{~cm}^{2}\right)$ were cleaned as follows ${ }^{[28]}$ : sonication in dichloromethane at $35^{\circ} \mathrm{C}$ for $15 \mathrm{~min}$, water-rinsing for 5 min, and then soaking in a cleaning bath at $80{ }^{\circ} \mathrm{C}$ for $15 \mathrm{~min}$. The cleaning solution was composed of $100 \mathrm{ml}$ of $96 \% \mathrm{H}_{2} \mathrm{SO}_{4}, 35 \mathrm{ml}$ of $35 \% \mathrm{H}_{2} \mathrm{O}_{2}$, and $65 \mathrm{ml}$ deionized water. The cleaned substrates were further rinsed in deionized water for $10 \mathrm{~min}$ and finally spin-dried. A diblock copolymer polystyrene-block-poly(ethylene oxide) denoted with $P(S-b-E O)$, with a total number average molecular weight $\mathrm{M}_{\mathrm{n}}=26.5 \mathrm{~kg} / \mathrm{mol}$, a weight ratio 3:1 (PS:PEO), and a polydispersity index $\left(\mathrm{M}_{\mathrm{w}} / \mathrm{M}_{\mathrm{n}}\right)$ of 1.05 was purchased from Polymer Source Inc. The polymer was dissolved in benzene at different concentrations and used for the coating step. The precleaned silicon substrates were coated by spin coating at $2500 \mathrm{rpm}$ for $30 \mathrm{~s}$. The polymer films were solvent-annealed inside an inverted beaker that fit a Petri dish containing $20 \mathrm{ml}$ benzene. The latter set-up is located inside an unsealed chamber that was placed in a hood (air flow at a speed of $0.2 \mathrm{~m} / \mathrm{s}$ ) with a controlled humidity of $30 \%$ at room temperature for $48 \mathrm{~h}$ (see Figure 1). The annealing time was selected such that the improvement in the alignment of the nano-structured polymer film is imparted without any excessive depletion of the polymer film by the vapor flow for a long time period. A proof of an air-flow parallel to the sample surface inside the annealing chamber was indicated by visualizing the flow of smoke stream of white ammonium chloride gas in the lab hood at a sash opening of about $20 \mathrm{~cm}$. A schematic diagram of the annealing set-up is shown in Figure 1.

Real-space and reciprocal-space film characterizations: Characterization of the microstructure, surface morphology and thicknesses of the DBC films was carried out with X- 
ray reflectometry $(\mathrm{XR})$, scanning electron microscopy (SEM), atomic force microscopy (AFM), and optical microscopy. The DBC film thickness was measured using XR before the solvent annealing step, using a laboratory Siemens D5000 X-ray source. The XR measurements were performed at a wavelength of $0.154 \mathrm{~nm}\left(\mathrm{Cu} \mathrm{K} \mathrm{\alpha}_{\alpha}\right.$ radiation) in air. The reflectivity data were analyzed within the Parratt approach ${ }^{[29]}$ using the Motofit software ${ }^{[30]}$ assuming a model which consists of a silicon substrate with its native oxide layer and a polymer layer on the top. Optical microscopy images with various magnifications between 2.5-100 times were collected for the polymer films using a Zeiss Axiotech 25H optical microscope equipped with a Hitachi KP-D50 CCD camera. Scanning electron micrographs were obtained with a LEO (model 1430) microscope operating at an acceleration voltage of 5 $\mathrm{kV}$ and a working distance $5 \mathrm{~mm}$. The $\mathrm{DBC}$ films were pre-sputter coated with a thin carbon film using argon plasma prior to the SEM measurements. Topographical and phase images of the DBC films were examined by AFM. The evaluation was performed across different surface areas (up to $3 \times 3 \mu \mathrm{m}^{2}$ ) under constant applied force conditions (non-contact mode) using an AFM AutoProbe CP Research Instrument. Micro-fabricated V-shaped silicon cantilevers with a spring constant $k=3.2 \mathrm{~N} / \mathrm{m}$ and a resonance frequency of approximately 80 $\mathrm{kHz}$ with a silicon conical tip of a typical radius of $10 \mathrm{~nm}$ were used.

Grazing-incidence X-ray diffraction (GIXRD): GIXRD experiments were performed at the beamline BW2 at HASYLAB (DESY, Hamburg) with a wavelength of $\lambda=0.124 \mathrm{~nm}^{[31]}$ The samples were mounted in a Kapton chamber which was flushed with $\mathrm{He}$ gas to minimize both sample damage and air scattering. The angle of incidence was chosen at $0.25^{\circ}$, and the scattered radiation was collected as a function of the angle $2 \theta$ in the film plane at a small exit angle $\alpha_{\mathrm{f}}$ using a scintillation detector. In this way, the intensity was recorded in dependence of the moment transfer $q_{\|}$in the surface plane, giving information on the in-plane crystalline structure. The selected incidence angle is much larger than the average critical angle of the polymer film $\left(\alpha_{c}=0.139^{\circ}\right)$ allowing bulk sensitive GIXRD measurements.

Grazing-incidence small-angle X-ray scattering (GISAXS) measurements: The GISAXS measurements were carried out at beamline $\mathrm{BW} 4^{[32]}$ of the DORIS III storage ring at HASYLAB (DESY, Hamburg). In GISAXS, the incoming X-ray beam impinges onto the sample surface at a small incidence angle $\alpha_{i}$, and the scattered signal is collected using a 2D detector. The selected wavelength was $\lambda=0.138 \mathrm{~nm}$. The pathway of the X-ray beam was evacuated and the beam was focused to the size of $30 \times 60 \mu \mathrm{m}^{2}$ using an assembly of 
beryllium lenses. The X-ray beam divergence in and out of the plane of reflection was set by high-quality entrance cross-slits. The two dimensional detector (MARCCD; 2048 x 2048 pixels) was placed at a distance of $L_{S D}=2 \mathrm{~m}$ behind the sample. For the experiments presented here, a rod like beam stop made of tantalum with a diameter of $1.5 \mathrm{~mm}$, mounted close to the detector, was used to protect the detector from the intensely reflected beam in the scattering plane. In addition, a point-like movable beam stop in front of the detector was used to block the specular reflection of the sample. The used incidence angle $\alpha_{i}=0.49^{\circ}$ is well above the critical angle of both, the DBC film and the substrate $\left(\alpha_{c}(\mathrm{PS})=0.154, \alpha_{c}(\mathrm{PEO})=\right.$ $\left.0.161^{\circ}, \alpha_{c}(\mathrm{Si})=0.22^{\circ}\right)$; therefore, the Yoneda peaks ${ }^{[33]}$ of both materials and specular peak are well separated on the 2D detector. At this angle of incidence, both the surface and bulk nanostructures of the polymer film are accessible. ${ }^{[34,35]}$ Structural information is obtained with horizontal slices $\left(q_{y}\right)$ of the 2D intensity distribution with $\Delta q=1.79 \times 10^{-3} \mathrm{~nm}^{-1}$.

\section{Results and discussion}

With the selection of $P(S-b-E O)$ a DBC with one amorphous and one crystalline block is addressed, which can exhibit an interplay between micro-phase separation and crystallization. Therefore such system offers the opportunity to install large areas of highly ordered crystalline nano-objects. The well known and simple mechanism ${ }^{[23]}$ of solvent evaporation via propagation of a well-ordered front from the surface into the bulk of the polymer film cannot explain the installed parallel cylinder morphology in our samples. Instead, an improved alignment of the DBC thin film morphology by an induced shearing effect via directional vapor flow is introduced.

\section{a) Influence of film thickness}

To work out the effect of film thickness, three different DBC film thicknesses $(30,65$, and 87 $\mathrm{nm})$ were studied and annealed in saturated benzene vapor under different solvent environmental conditions.

With respect to the polymer film thickness, different micro-phase separation morphologies and crystallization behaviors are observed. For a thin DBC film $(30 \mathrm{~nm})$, small square-shaped crystals dispersed in the film are obtained (see Figure 2a). The flat square-shaped crystals are formed by a reorganization process of the film so that PS chains flip to the top of the PEO crystals during the crystallization step. The crystallization process of the PEO in the thin PEO containing DBC films has previously been observed ${ }^{[36,37]}$ and dewetted polymer film were also obtained (see Figure 2a). In contrast, for the thicker film (87 nm), aggregates of 
crystalline PEO (Figure 2b) grow from a sheaf-like central structure and behave similar to those known for spherulite crystals of pure PEO films. ${ }^{[38]}$ Both, the fully crystallized thick films $(87 \mathrm{~nm})$ and the square-shaped crystal-forming polymer thin film $(30 \mathrm{~nm})$ do not show any distinct ordered nano-structures caused by micro-phase separation. Neither SEM and AFM nor GISAXS show a well ordered nano-scale structure at the surface or in the bulk of the $P(S-b-E O)$ films.

The crystallization behavior of the DBC films exposed to vapor flow is thus thickness dependent. Generally, it is assumed that the crystallization of DBCs (composed of one semicrystalline block and one amorphous block) proceeds via chain aggregation of the crystallizable block (PEO) in a limited manner due to constrains imparted by the linked amorphous block. ${ }^{[39,40]}$ However, the high mobility of both polymer blocks (PEO and PS) in a good solvent (solubility parameter of benzene, $\delta=18.8 \mathrm{MPa}^{1 / 2}$ ) for both $\mathrm{PS}\left(\delta=18.6 \mathrm{MPa}^{1 / 2}\right)$ and PEO ( $\delta=20.0 \mathrm{MPa}^{1 / 2}$ ) blocks, during the solvent annealing step allows the PEO chains to crystallize, forming hierarchically ordered structures over a large range of length scales from the crystal unit cell and nano-meter sized crystallites up to hundreds of microns for spherulites. Such high mobility of both blocks in a good solvent enables unhindered chain folding by the strong driving force of the crystallization process. The crystallization that results in spherulites formation, for instance, is not influenced by the PS confined environment. It has been recently reported $^{[41]}$ that the crystallization growth in cylindrical geometries of microphase separated DBCs films can lead to the destruction of the mesophase pattern, allowing for a break-up crystallization. Therefore, the crystallization forces are incomparably stronger than the micro-phase separation process involved in the self-assembly of amorphous DBCs films. ${ }^{[42]}$

For an intermediate value of the film thickness $(65 \mathrm{~nm})$, however, a partially crystalline polymer film with surface terraces consisting of finger-like crystalline domains is observed as revealed from the real-space characterizations including optical microscopy (see Figure 2c), scanning electron microscopy (see Figure 3), and atomic force microscopy (see Figure 4). Upon benzene vapor treatment, polystyrene domains sandwiched between crystalline PEO domains in $P(S-b-E O)$ are observed in areas between the large non-regular crystalline domains of the polymer film. The striking observation in the current study is the formation of highly oriented cylinder morphology (see Figure 4a,b) via benzene treatment of the polymer film (with thickness $65 \mathrm{~nm}$ ) with the vapor flow parallel to the sample surface. In the next section the installation of this special surface structure is discussed. 


\section{b) Installation of highly ordered micro-phase separation structure}

The benzene vapor treatment of the DBC samples (necessary to achieve the highly ordered nanostructures) was performed in a controlled humidity of $30 \%$ at room temperature with a vapor flow speed of $0.2 \mathrm{~m} / \mathrm{s}$ inside a fume hood (see Figure 1). A random orientation of PEO cylinders (in a PS matrix) parallel to the film surface is observed (see Figure 4a) for the same sample exposed to benzene vapor without using the flow effect (fume hood is turned off). Parallel vapor flow speed of $0.2 \mathrm{~m} / \mathrm{s}$ in the unsealed annealing set-up reveals that the cylinder domains are preferentially oriented by the directional flow of benzene vapor during the annealing step. The morphologies with and without the vapor flow in an unsealed vapor annealing set-up were reproducible.

The structural characteristic in terms of dominant length scales is obtained from GISAXS. ${ }^{[35]}$ The GISAXS patterns of the polymer films (thickness $65 \mathrm{~nm}$ ) with and without the parallel vapor flow to the sample surface are presented in Figure 5. A similar lateral spacing $(31 \mathrm{~nm})$ between the two neighboring PEO cylinders is observed irrespective of the vapor flow while a highly orientated morphology is imparted, as revealed from both the second order scattering rod in the GISAXS pattern (see Figure 5) and the AFM data (see Figure 4a,b). A second order rod-like peak at the position $2 \mathrm{q}^{*}$ in the GISAXS data confirms the highly ordered polymer morphology of PEO cylinders being parallel in orientation with respect to the surface plane (see Figure 6). We attributed the highly oriented morphology of the nano-structured polymer film to vapor flow shearing effect. Interestingly, an increase of the vapor speed (created by increasing the suction fan speed of the operated hood) led to a depletion of the polymer film in the benzene vapor surrounding (see Figure 2c).

For the polymer films treated at the benzene vapor flow speed of $0.2 \mathrm{~m} / \mathrm{s}$, it was possible to obtain large scale ordered surface areas. Typically these areas of highly-oriented parallel PEO nano-cylinders are $7 \mu \mathrm{m}$ in size. Domains of different orientations coexists (see Figure 4c) and are separated by break-out large irregular crystalline areas. Microscopic images (see Figure 2c) show that oriented macroscopic structures are present at the edge of the samples and around the deformed large crystallites. The polymer film (thickness $65 \mathrm{~nm}$ ) under high vapor flow deforms evenly in one direction where no crystals exist, while at areas with large crystallites the deformation takes a circular shape (see Figure 2c).

\section{c) Interplay with crystalline structure}

To get a better understanding of the interplay between highly ordered micro-phase separation structure and crystalline structure, GIXRD experiments were performed on the $\mathrm{P}(\mathrm{S}-b-\mathrm{EO})$ 
film of intermediate thickness $(65 \mathrm{~nm})$. The in-plane GIXRD data of the vapor-annealed polymer film show only one Bragg reflection peak, indicating that the film is not fully crystallized (see Figure 6). The high-resolution scan of this Bragg peak is shown as an inset in Figure 6. Based on the monoclinic PEO crystal, the unit cell has four helical molecules along the $c$-axis (chain axis). ${ }^{[43]}$ Several reflections in the reciprocal space have been previously identified by rotating a highly ordered PEO crystal around its $c$-axis. ${ }^{[43,44]}$ The strongest (120) reflection has an angle of $90^{\circ}$ with respect to the c-axis and a d-spacing of $0.463 \mathrm{~nm}$. The GIXRD Bragg peak we have observed in case of the $65 \mathrm{~nm}$ thick film is thus assigned for the (120) plane of an oriented PEO monoclinic crystal and suggests that the c-axis of the unit cell is oriented perpendicular to the film surface. ${ }^{[37]}$ The chains are packed regularly together along the c-axis and perpendicular to the cylinder axis (as shown schematically in Figure 6). Recently, it has been reported ${ }^{[37,43]}$ that for lamella forming $P(S-b-E O)$ diblock copolymer the crystal orientation (the $c$-axis of the PEO crystals) can change from random to perpendicular, then to inclined, and finally to parallel with respect to the lamellar surface, depending on the crystallization temperature. With respect to the surface plane in our sample, the perpendicular orientation of the PEO crystalline stems should be considered as an average orientation due to the tethering of the confined PEO block at the interface. A perfectly oriented crystal cannot be assumed. For the PEO nano-domains (cylinders) embedded inside the PS matrix in our polymer film, the crystallization is relatively highly oriented (see Figure 6) and approaches sizes that are of the order of the cylinder diameter (confining size). The apparent mean PEO crystal size along [hkl] direction in the 2D PS-confinement is calculated using the Scherrer equation. The resulting mean PEO crystal size of $7.8 \mathrm{~nm}$ perfectly matches the full diameter of the PEO cylinder $8.8 \mathrm{~nm}$ domains, indicating high crystallinity within the PEO cylindrical domains. Thus, the crystals grow along the cylinder axis and favor a perpendicular orientation of the PEO crystal stem with respect to the surface plane as schematically depicted in Figure 6. The absence of any out-of-plane GIXRD reflection is an additional proof of preferential PEO stem direction perpendicular to the film surface for all different PEO crystallites including the break-out crystals.

A possible mechanism for the formation of a crystalline nano-cylinder morphology embedded into an amorphous polymer matrix can be proposed via the reorganization of the whole polymer film. Upon solvent vapor treatment, the formation of macroscopic crystallite centers with extended finger-like crystalline domains may migrate parallel to the surface forming the mesoscopic structure. Finger-like branched patterns were previously observed for low molecular weight $P(S-b-E O)$ block copolymer thin films in quasi-2d geometry. ${ }^{[45]}$ The 
directional solidification of semicrystalline PEO during the spherulite formation has a radial direction from the nucleation center. Such directional crystallization may provide an alignment along the gradient as the nucleation is suppressed ahead of the solidification front. In the direction of higher mobility of the crystals, the association of neighboring growing fingers is much less as a result of low availability of free molecules and hence, PEO cylinders in the nano-scale size are formed and intercalated between two domains of PS blocks.

Our results, however, do not support the proposed mechanism via an extended crystalline finger for the formation of nano-structured polymer morphology. AFM measurements at the boundaries between the large macroscopic crystallites and the nano-cylinders locations (see Figure 4d) do not show a smooth transition from the large crystalline centers into the nanometer size-ordered structures. In other words, no crystalline finger-like domains extend into the nano-cylinder domains.

Prior to the vapor annealing stage, a cylinder-forming phase-separated polymer film via the repulsion interaction of incompatible polymer blocks (by micro-phase separation) has also been observed following the spin coating step. Therefore, we assume that during the vapor treatment some of the phase-separated PEO cylindrical domains (see Figure 4) are not involved in the macroscopic polymer mobility process (hence the formation of irregular large crystalline areas) and instead undergo local crystallization on a length scale that is superposed by the mesoscopic structures.

\section{Conclusion}

The formation of the highly oriented crystalline cylinder morphology under the influence of directional parallel benzene vapor flow produces a preferential orientation in the polymer morphology of the $P(S-b-E O)$ DBC. It reveals that with the help of the directional vapor flow a crystallization-induced morphology alignment in DBC thin films is possible. The highly parallel oriented cylinder morphology of the polymer film is explained by the vapor flow induced shearing effect rather than an initial order front propagation. As compared to DBC morphologies achievable with DBCs with two amorphous blocks, the domain size however is still limited to several micrometers due to the interplay with crystallization. Although we focus only on one particular diblock copolymer, namely $P(S-b-E O)$, this might be a general phenomenon in semicrystalline diblock copolymer thin films. 
Acknowledgments: This research was financially supported by DFG in the priority program for nano- and microfluidics SPP1164, Grant No. MU1487/2. Beamtimes at HASYLAB, BW4 and BW2 are acknowledged. We are grateful to Dr. Carsten Deiter for his help at BW2 beamline. 
References:

[1] I. W. Hamley, Angewandte Chemie-International Edition 2003, 42, 1692.

[2] R. A. Segalman, Mat Sci Eng R 2005, 48, 191.

[3] I. W. Hamley, Nanotechnology 2003, 14, R39.

[4] R. S. Krishnan, M. E. Mackay, P. M. Duxbury, A. Pastor, C. J. Hawker, B. Van Horn, S. Asokan, M. S. Wong, Nano Lett 2007, 7, 484.

[5] W. Tjandra, J. Yao, P. Ravi, K. C. Tam, A. Alamsjah, Chem Mater 2005, 17, 4865.

[6] T. Thurn-Albrecht, J. Schotter, C. A. Kastle, N. Emley, T. Shibauchi, L. KrusinElbaum, K. Guarini, C. T. Black, M. T. Tuominen, T. P. Russell, Science 2000, 290, 2126.

[7] J. Q. Lu, S. S. Yi, Langmuir 2006, 22, 3951.

[8] C. Minelli, C. Hinderling, H. Heinzelmann, R. Pugin, M. Liley, Langmuir 2005, 21, 7080 .

[9] S. Horiuchi, T. Fujita, T. Hayakawa, Y. Nakao, Langmuir 2003, 19, 2963.

[10] M. Adachi, A. Okumura, E. Sivaniah, T. Hashimoto, Macromolecules 2006, 39, 7352.

[11] J. Fu, X. S. Fen, Y. C. Han, C. Y. Pan, Y. M. Yan, B. Y. Li, Macromol Rapid Comm 2003, 24, 487.

[12] Y. N. Xia, E. Kim, X. M. Zhao, J. A. Rogers, M. Prentiss, G. M. Whitesides, Science 1996, 273, 347.

[13] S. R. Quake, A. Scherer, Science 2000, 290, 1536.

[14] J. Schmitt, G. Decher, W. J. Dressick, S. L. Brandow, R. E. Geer, R. Shashidhar, J. M. Calvert, Adv Mater 1997, 9, 61.

[15] J. Y. Cheng, C. A. Ross, E. L. Thomas, H. I. Smith, G. J. Vancso, Adv Mater 2003, 15, 1599.

[16] Z. Y. Di, D. Posselt, D. M. Smilgies, C. M. Papadakis, Macromolecules 2010, 43, 418.

[17] S. O. Kim, H. H. Solak, M. P. Stoykovich, N. J. Ferrier, J. J. de Pablo, P. F. Nealey, Nature 2003, 424, 411.

[18] S. M. Park, G. S. W. Craig, Y. H. La, P. F. Nealey, Macromolecules 2008, 41, 9124.

[19] L. Tsarkova, A. Knoll, G. Krausch, R. Magerle, Macromolecules 2006, 39, 3608.

[20] E. Gattiglia, A. Turturro, D. Ricci, A. Bonfiglio, Macromol Rapid Comm 1995, 16, 919.

[21] D. E. Angelescu, J. H. Waller, R. A. Register, P. M. Chaikin, Adv Mater 2005, 17, 1878.

[22] A. Böker, H. Elbs, H. Hansel, A. Knoll, S. Ludwigs, H. Zettl, A. V. Zvelindovsky, G. J. A. Sevink, V. Urban, V. Abetz, A. H. E. Müller, G. Krausch, Macromolecules 2003, 36, 8078.

[23] S. H. Kim, M. J. Misner, T. Xu, M. Kimura, T. P. Russell, Adv Mater 2004, 16, 226.

[24] J. Peng, Y. C. Han, W. Knoll, D. H. Kim, Macromol Rapid Comm 2007, 28, 1422.

[25] J. Peng, Y. H. Wei, H. F. Wang, B. Y. Li, Y. C. Han, Macromol Rapid Comm 2005, 26, 738 .

[26] C. M. Papadakis, Z. Y. Di, D. Posselt, D. M. Smilgies, Langmuir 2008, 24, 13815.

[27] P. Lightfoot, M. A. Mehta, P. G. Bruce, Science 1993, 262, 883.

[28] P. Müller-Buschbaum, Eur Phys J E 2003, 12, 443.

[29] L. G. Parratt, Phys Rev 1954, 95, 359.

[30] A. Nelson, J Appl Crystallogr 2006, 39, 273.

[31] O. Bunk, M. M. Nielsen, J Appl Crystallogr 2004, 37, 216.

[32] S. V. Roth, R. Döhrmann, M. Dommach, M. Kuhlmann, I. Kröger, R. Gehrke, H. Walter, C. Schroer, B. Lengeler, P. Müller-Buschbaum, Rev Sci Instrum 2006, 77, 085106.

[33] Y. Yoneda, Phys Rev 1963, 131, 2010. 
[34] T. Salditt, T. H. Metzger, J. Peisl, B. Reinker, M. Moske, K. Samwer, Europhys Lett 1995, 32, 331.

[35] P. Müller-Buschbaum, Analytical and Bioanalytical Chemistry 2003, 376, 3.

[36] P. Yang, Y. C. Han, Macromol Rapid Comm 2008, 29, 1614.

[37] C. Darko, I. Botiz, G. Reiter, D. W. Breiby, J. W. Andreasen, S. V. Roth, D. M. Smilgies, E. Metwalli, C. M. Papadakis, Physical Review E 2009, 79, 041802.

[38] X. B. Huang, Y. W. Huang, L. Zhu, X. Z. Tang, Polym. Compos. 2008, 29, 1297.

[39] G. Reiter, G. Castelein, P. Hoerner, G. Riess, A. Blumen, J. U. Sommer, Phys Rev Lett 1999, 83, 3844.

[40] G. Reiter, G. Castelein, P. Hoerner, G. Riess, J. U. Sommer, G. Floudas, Eur Phys J E 2000, 2, 319.

[41] C. Vasilev, G. Reiter, S. Pispas, N. Hadjichristidis, Polymer 2006, 47, 330.

[42] I. W. Hamley, Adv Polym Sci 1999, 148, 113.

[43] L. Zhu, S. Z. D. Cheng, B. H. Calhoun, Q. Ge, R. P. Quirk, E. L. Thomas, B. S. Hsiao, F. J. Yeh, B. Lotz, J Am Chem Soc 2000, 122, 5957.

[44] P. Huang, Y. Guo, R. P. Quirk, J. J. Ruan, B. Lotz, E. L. Thomas, B. S. Hsiao, C. A. Avila-Orta, I. Sics, S. Z. D. Cheng, Polymer 2006, 47, 5457.

[45] G. Reiter, L. Vidal, Eur Phys J E 2003, 12, 497. 


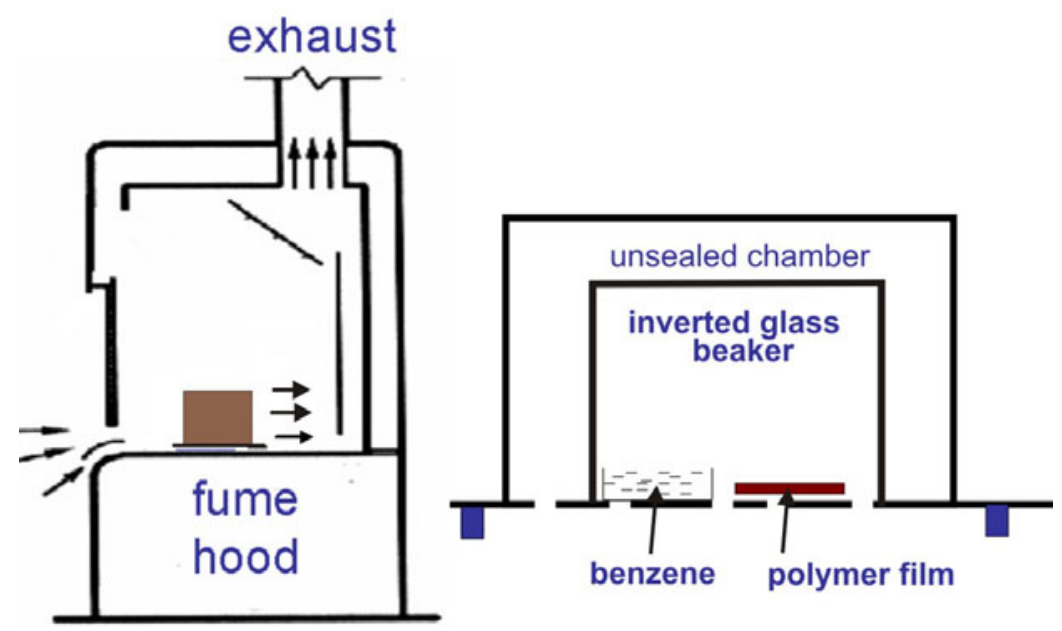

Figure 1. Schematic diagram showing the benzene vapor annealing set-up.

(a)
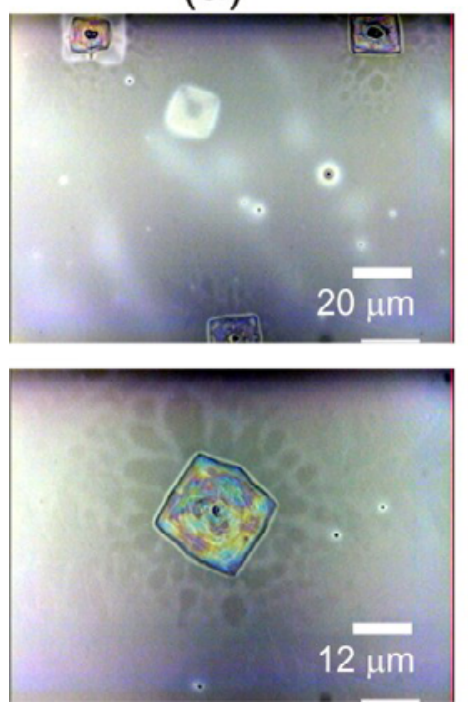

(b)
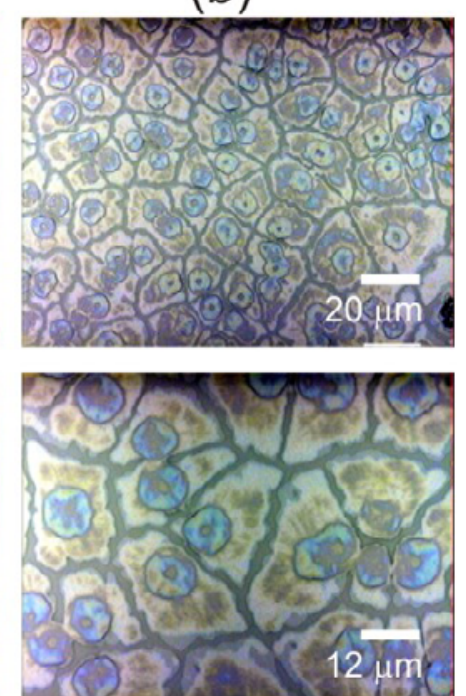

(c)
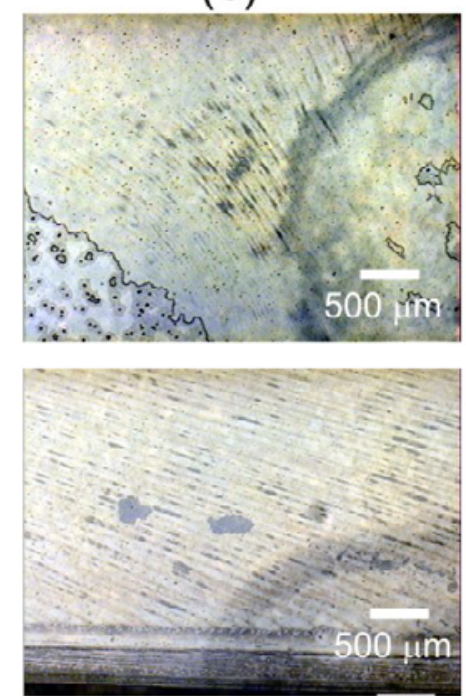

Figure 2. Optical microscope images of films with (a) $30 \mathrm{~nm}$ thickness, showing squareshaped crystals, (b) $87 \mathrm{~nm}$ thickness, showing spherulite crystals, and (c) $65 \mathrm{~nm}$ thickness prepared under high vapor flow speed, showing a depletion of the film at the edge and around the crystalline domains. 

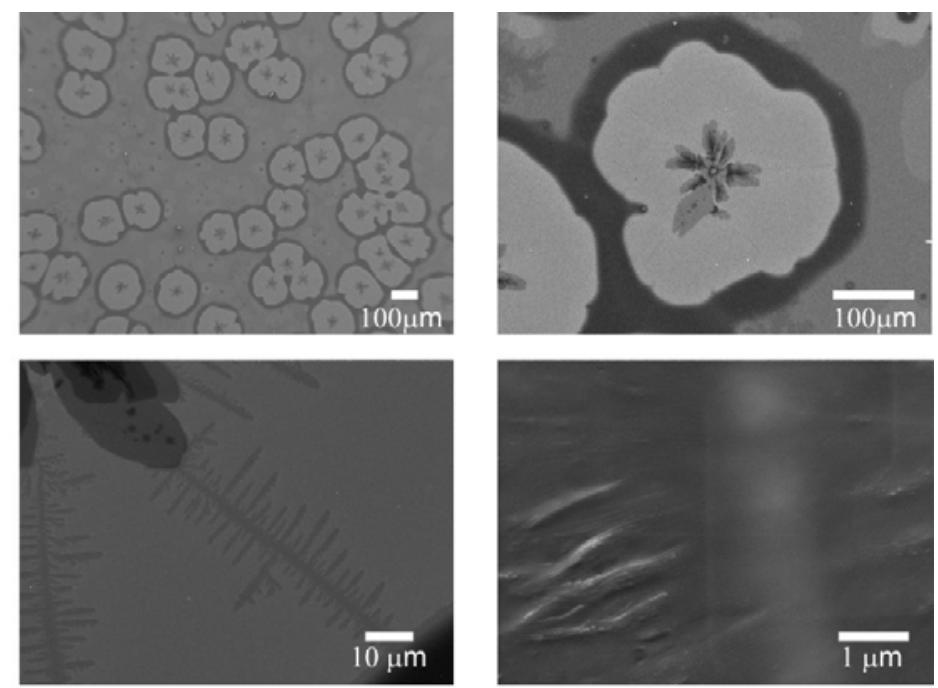

Figure 3. Scanning electron microscopy images of the films with $65 \mathrm{~nm}$ thickness after treatment in parallel benzene vapor flow: Different magnifications show the partially crystalline film with finger-like structures.
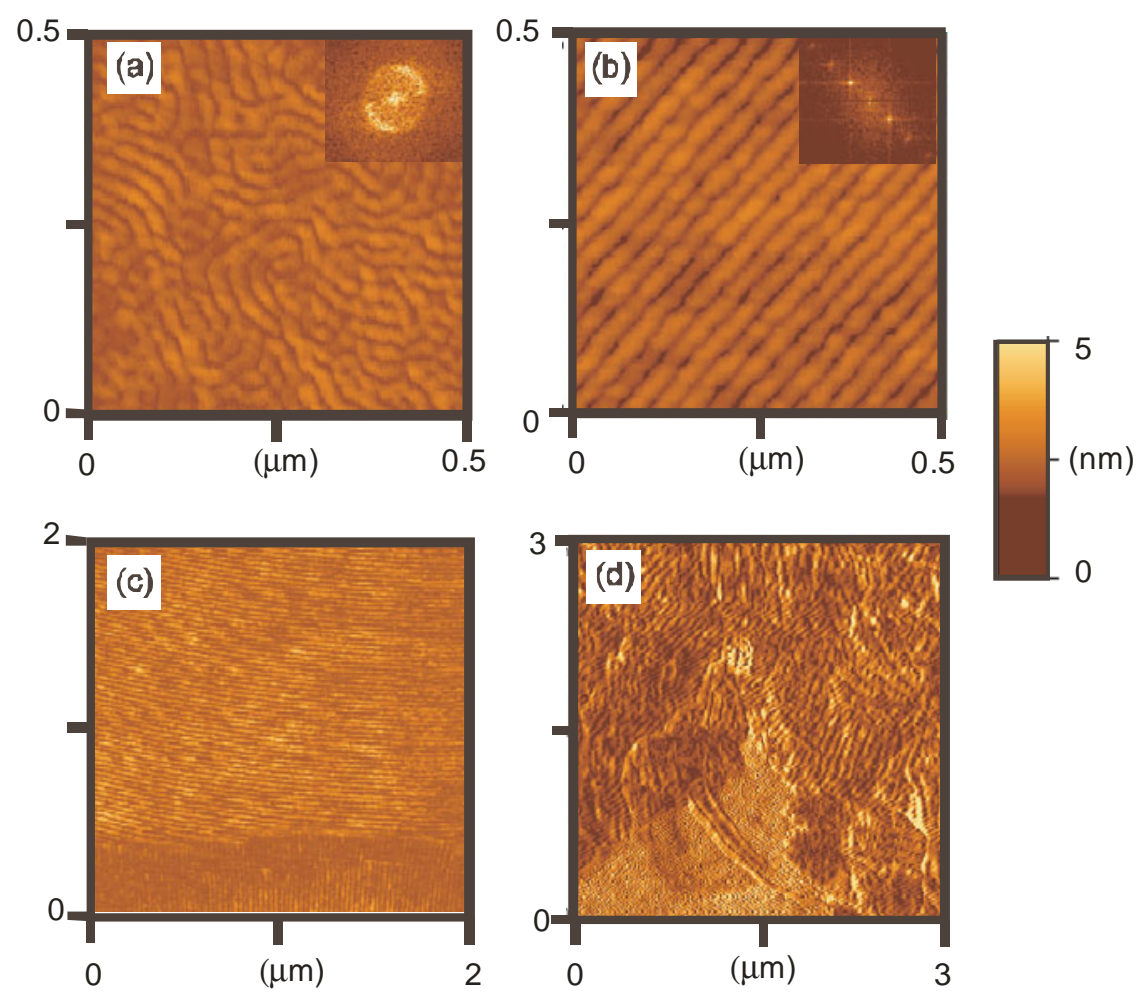

Figure 4. AFM images of the $\mathrm{P}(\mathrm{S}-b$-EO) film with $65 \mathrm{~nm}$ thickness after benzene vapor treatment without (a) and with vapor flow (b, c, and d) at speed of $0.2 \mathrm{~m} / \mathrm{s}$. (b) highly oriented parallel cylinders are formed upon vapor flow shearing. (c) Cylindrical domains have high directional orientation and meet where the circular vapor flow exists. (d) No smooth transition from the large ill-defined crystalline domains into the well-defined nano-structured film support the crystallization superposition of the micro-phase separated cylinder domains. 

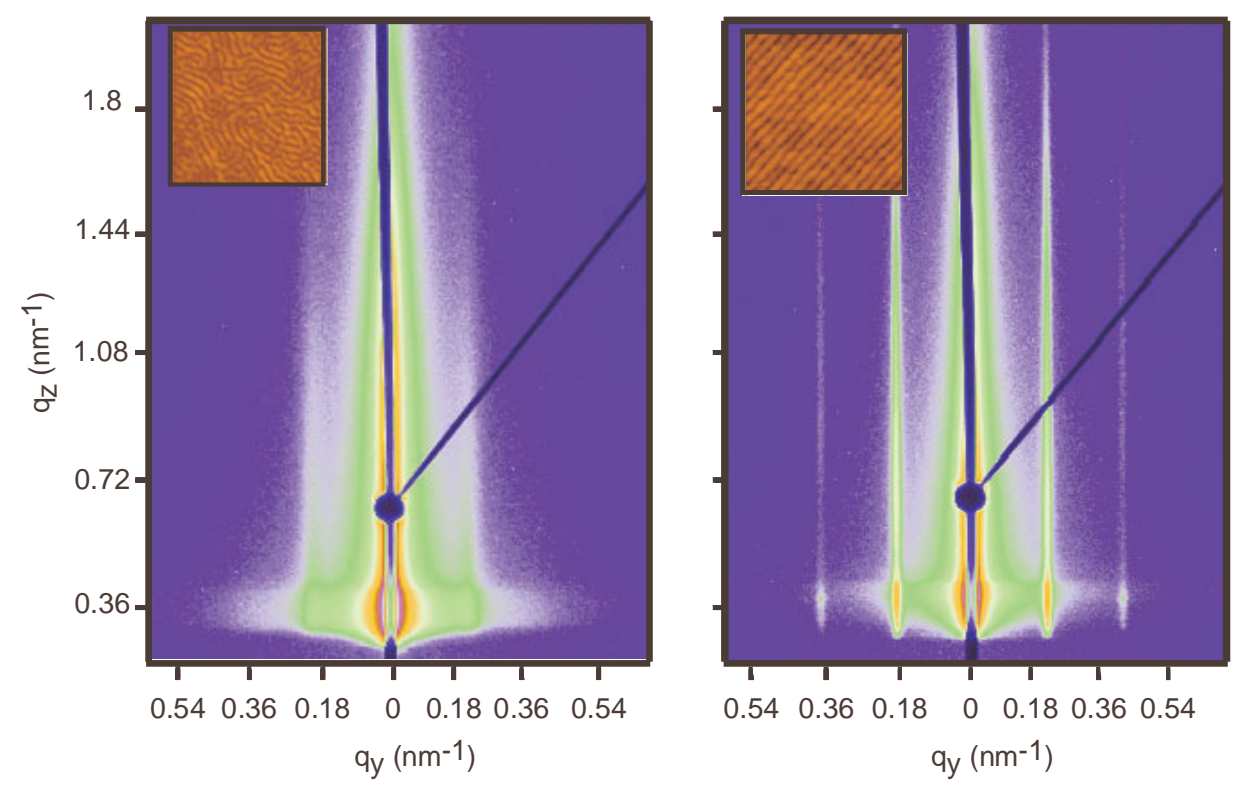

Figure 5. GISAXS 2d patterns of films with $65 \mathrm{~nm}$ thickness (left) without and (right) with applied directional vapor flow: A second order rod-like peak at the position $2 q^{*}$ in the GISAXS pattern (right) confirms the highly oriented cylinder morphology being aligned parallel with respect to the surface plane upon directional benzene vapor flow.
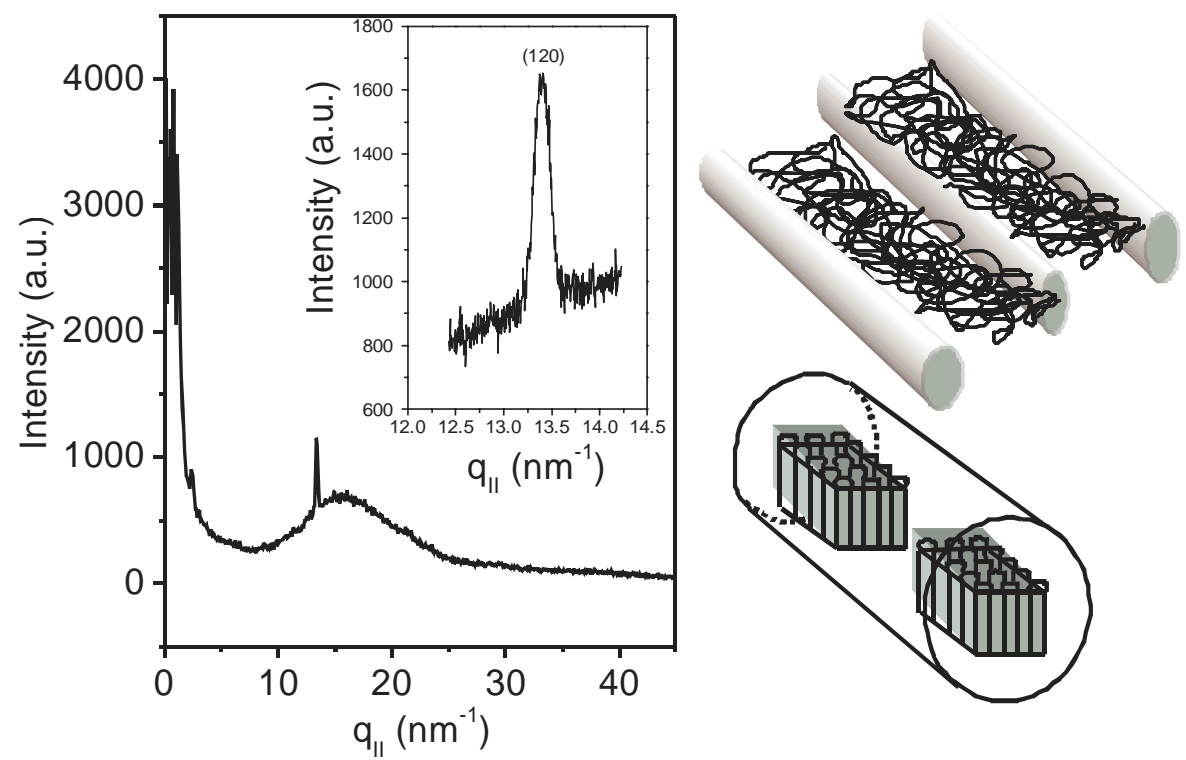

Figure 6. In-plane GIXRD intensity profile of the film with $65 \mathrm{~nm}$ thickness after treatment in parallel benzene vapor flow. The high resolution scan of the single Bragg reflection (120) peak is shown in the inset. A schematic drawing shows the orientation of the homogeneous crystal within the confined cylinder. 


\section{The table of contents entry}

An induced large scale alignment of the parallel cylinder morphology of the polystyreneblock-polyethylene oxide $\mathrm{P}(\mathrm{S}-b$-EO) thin film via treatment in a saturated benzene vapor that flows parallel to the film surface is realized. The enhanced alignment in the vapor-flow is attributed to a vapor-flow induced shearing effect of the polymer film. The semicrystalline PEO block forms cylinders that initiate a growth rate with a favorable perpendicular orientation of the PEO crystalline stems with respect to the cylinder axis.

Ezzeldin Metwalli, Jan Perlich, Weinan Wang, Alexander Diethert, Stephan V. Roth, Christine M. Papadakis, and Peter Müller-Buschbaum

\section{ToC Figure}

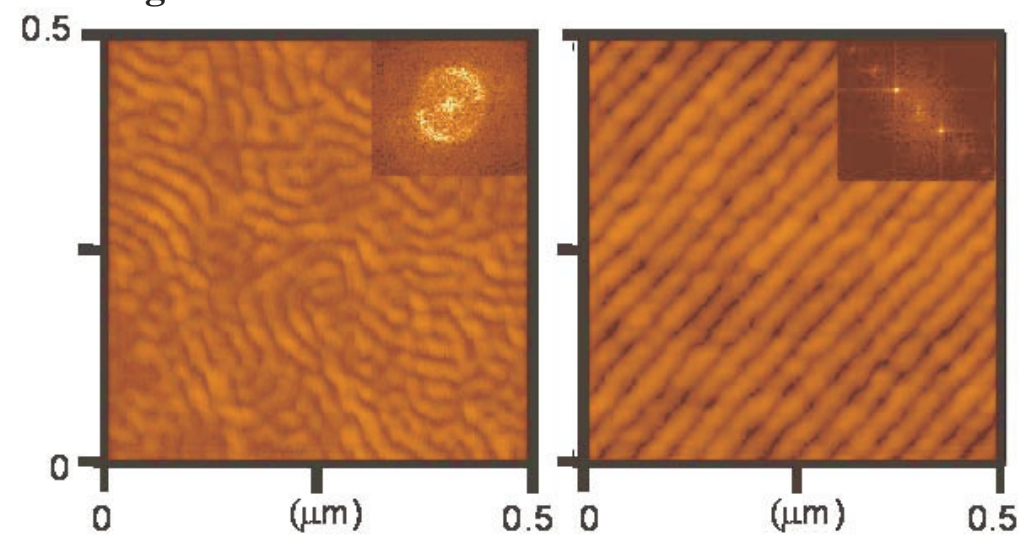

